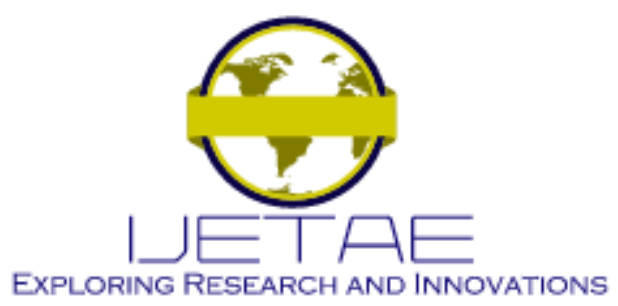

International Journal of Emerging Technology and Advanced Engineering

Website: www.ijetae.com (E-ISSN 2250-2459, Scopus Indexed, ISO 9001:2008 Certified Journal, Volume 11, Issue 12, December 2021)

\title{
The Method for Identification of Radars Measurements of Nearby Objects Tracking
}

\author{
Hennadii Khudov ${ }^{1}$, Igor Ruban ${ }^{2}$, Hennadii Pievtsov $^{3}$, Oleksandr Makoveichuk $^{4}$, Oleh Popkov ${ }^{5}$, Danylo Shabanov ${ }^{6}$, \\ Yurii Baranov $^{7}$, Yuriy Solomonenko ${ }^{8}$, Vitalii Kryvosheiev ${ }^{9}$, Rostyslav Khudov ${ }^{10}$ \\ ${ }^{1,3,8}$ Ivan Kozhedub Kharkiv National Air Force University, Ukraine, Kharkiv, 61023 \\ ${ }^{2,4}$ Kharkiv National University of Radio Electronics, Ukraine, Kharkiv, 61166 \\ ${ }^{5}$ Central Scientific Research Institute of Armament and Military Equipment of the Armed Forces of Ukraine, Ukraine, \\ Kyiv, 03049 \\ ${ }^{6}$ State Scientific Research Institute of Armament and Military Equipment Testing and Certification, Ukraine, Chernihiv, 14033 \\ ${ }^{7}$ Hetman Petro Sahaidachnyi National Army Academy, Ukraine, Lviv, 79026 \\ ${ }^{9}$ National Defense University of Ukraine named after Ivan Cherniakhovskyi, Ukraine, Kyiv, 01001 \\ ${ }^{10}$ V. N. Karazin Kharkiv National University, Ukraine, Kharkiv, 61022
}

\begin{abstract}
The paper proposes the method for identification of radars measurements of nearby objects tracking. The method for identification of radars measurements of nearby objects tracking, in contrast to the known ones, assumes the next. Solving the optimization problem of searching for a hypothesis of joint identification of objects and measurements. The solution is optimal according to Bayes' criterion. The solution of the optimization problem of joint identification of objects and measurements is reduced to the assignment problem with the subsequent choice of a hypothesis. The choice of hypothesis is presented in the form of an identification matrix. The presence of background objects in the viewing area is taken into account. The procedure for enumerating hypotheses is rather cumbersome even with a small number of objects (10-12 objects). Therefore, the subject of further research is to find ways to reduce the computational costs of solving the optimization problem.
\end{abstract}

Keywords - method, identification, radar, measurement, nearby object, tracking, hypothesis

\section{INTRODUCTION}

The problem of identifying nearby objects arises in many different situations. As a rule, there are several air objects in the air space at the same time. In this case, it is necessary to separate and identify these objects. The identification of radar measurements from two airborne objects is shown in Fig. 1 [1].

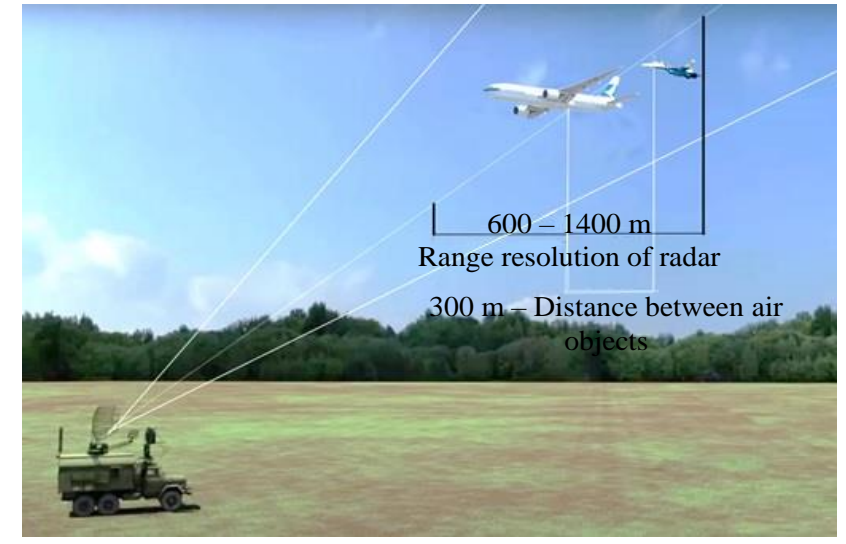

Fig. 1 Identification of radar measurements from two air objects [1]

The problem of identifying nearby objects is especially relevant for monitoring the space by the radars. Currently, more than 37,000 users of space services from 110 countries of the world are officially registered, and about 70 countries have their own spacecraft [2]. At the same time, about 950 spacecraft operate in orbits around the Earth, of which $36 \%$ are in low orbits, $6 \%$ are in medium orbits, $48 \%$ are in geostationary orbit, and $10 \%$ are in highly elliptical and ultrahigh orbits [2].

Along with the expansion of the exploration of nearearth space, its man-made debris is also increasing, to which attention was not immediately drawn. 


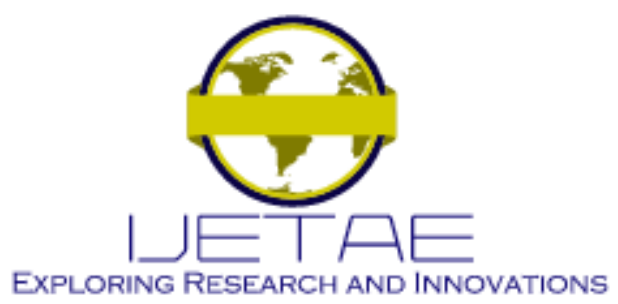

International Journal of Emerging Technology and Advanced Engineering

Website: www.ijetae.com (E-ISSN 2250-2459, Scopus Indexed, ISO 9001:2008 Certified Journal, Volume 11, Issue 12, December 2021)

For a very long time, the prevailing opinion about the immensity and boundlessness of space, about the idea of outer space as a "bottomless barrel" into which you can dump garbage in any quantity with impunity. All this has now led to a catastrophic state of technical debris in nearearth space.

The reduction in the mass and dimensions of modern spacecraft, along with the need to minimize the cost of launching satellites, made the concept of launching the "one launch - one satellite" type invalid. The accumulated experience now makes it possible to widely use the concept of launching the type "one launch - N satellites", which significantly complicates the solution of the problem of information support for the post-launch period of spacecraft commissioning. An additional complication is the fact that space objects can be in orbits with similar parameters, or even in orbits that differ, and only slightly, by only one or two parameters. Such situations are possible when space objects are accompanied by a dense stream, when launched by compact groups of spacecraft, after the destruction of the spacecraft or the last stage of the launch vehicle. In this case, the problem arises to resolve the objects moving in a group and to avoid their confusion. As a spacecraft representing a dense flow, one can give an example: spacecraft IKONOS-2, SPOT-5, LANDSAT-7 and SPOT-5 launch vehicle - IDEFIX / ARI 4 R [3-4].

One of the most difficult problems in the theory and technology of radar information processing is the joint tracking of several objects moving at short distances from each other. This takes place when objects trajectories are crossed or when objects are flown by a group. In practice, traditionally, the solution to the classification problem is reduced to calculating gates and identifying vectors of radio engineering measurements for each object. In the case of a dense stream of objects (for example, the output of several satellites or the initial stage of launch), the solution of the identification problem by the indicated methods is difficult. The tracking gates overlap with each other and the obtained marks can be identified with several trajectories. The task becomes even more complicated if some marks are missed or false marks have entered the strobe caused by natural (tracking of background objects) or artificial interference. At the same time, the requirements for the quality and efficiency of the problem being solved are often extremely high.

Thus, the task of developing a method for identification of radars measurements of nearby objects tracking is urgent.

\section{LITERATURE REVIEW AND PROBLEM STATEMENT}

The problems of synthesizing trajectory processing algorithms in the multipurpose case have been considered in many works. Known algorithms and methods for identifying coordinate information can be classified into Bayesian and non-Bayesian, strobe and non-strobe, with sequential and parallel measurements.

Fengler A. [5] notes that the simplest non-Bayesian approach is that for each mark hitting the gate, the likelihood coefficient of its belonging to the tracked trajectories is calculated. To continue the trajectory, one of them is selected for which this coefficient takes the maximum value. When using the statistical distance of the elevation from the center of the gate as a measure of likelihood, the elevation closest to the center of the gate is selected to continue the trajectory.

Tu. G et. Al. [6] note that another non-Bayesian approach could be the branching method. The essence of this method is as follows. When more than one mark is received in the strobe, the trajectory forks, and a likelihood function is calculated for each fork. Further, by comparing the likelihood function with the threshold, variants (branches) that have not passed the threshold test are discarded. The rest of the options continue. The main problem in this case is the choice of the threshold value, as well as the significant computational complexity.

The methods [5-6] can be classified as non-Bayesian sequential strobe methods. These methods have proved to be effective when tracking a small number of objects, provided there is a large distance between objects, a low level of interference (a small number of false marks), and high thresholds in primary processing devices. The methods [5-6] work at distance of up to 250-300 km with a distance between objects of 1-2 km.

It is known that in mathematical statistics Bayesian methods are considered the best classification methods. In this case, the classification problem is posed as the problem of testing a multi-alternative hypothesis. To solve it, it is necessary to generate a vector of statistics with the number of elements equal to the number of objects in the catalog. In the future, as a solution, the object is selected, the statistics of which is the best from the point of view of a given quality criterion, as which any criterion from the criteria of the Bayesian group can be selected [7-8]: maximum likelihood; maximum of a posteriori information; minimum average risk; the maximum average payoff. 


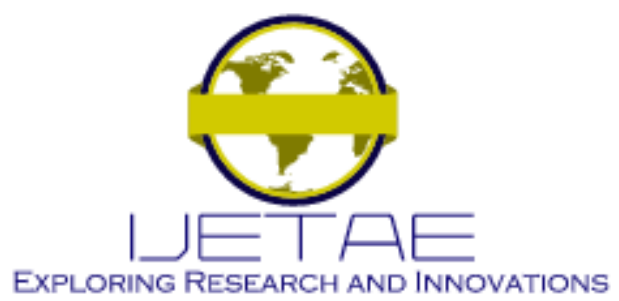

International Journal of Emerging Technology and Advanced Engineering

Website: www.ijetae.com (E-ISSN 2250-2459, Scopus Indexed, ISO 9001:2008 Certified Journal, Volume 11, Issue 12, December 2021)

When solving the problem of processing location information, a priori and a posteriori Bayesian classifiers are distinguished [9-10]. In a priori Bayesian classifiers, statistics of hypotheses about the membership of measurements are calculated based on the parameters of the trajectories obtained in the previous review. The classification process is preceded by the process of evaluating the trajectory parameters based on the data of the current survey. Subsequently, a posteriori estimates of the trajectory parameters are substituted into the decision rule of the Bayesian classifier [9-10].

For the first time, the idea of the method of a posteriori Bayesian classifier was proposed by Bar-Shalom [11], and later the method was generalized to the multipurpose case [12-13]. The algorithm for tracking close trajectories corresponding to this method was called the method of joint probabilistic data identification [12-13].

Authors [14] propose a method based on the optimal Bayesian approach to solving the problem of identifying new marks. The method assumes the calculation at each step of observation of the posterior probabilities of possible options for constructing the trajectory from the data of all observation cycles. The variant with the maximum posterior probability of realization is chosen as the optimal one. The total number of options for constructing trajectories in this case can be calculated in accordance with (1):

$$
I_{N}=\prod_{j=1}^{Z}\left(1+k_{j}\right) .
$$

where $k_{j}$ is the number of marks sampled in the strobe at the $\mathrm{j}$-th step of observation.

LeRoy T.E. [15] note that with a low probability of the presence of false marks, large distances between objects in relation to measurement errors, the methods of a priori and a posteriori Bayesian data identification give absolutely the same results. In the presence of compact groups of closely spaced objects, from the point of view of classification quality indicators, the posterior method [15] looks preferable. However, it requires an order of magnitude increase in the amount of computation for its implementation. The disadvantage of a priori Bayesian classifiers is the need to calculate a vector of statistics with the number of elements equal to the number of catalog objects. Moreover, their complexity linearly depends on the number of objects in the catalog used and on the total number of objects.
The number of hypotheses can be reduced by discarding that part of them that have a small posterior probability. Then you can combine hypotheses for which the same measurements are included in the last $n$ measures. In [16] a quasi-optimal Bayesian method is proposed, suitable for practical implementation, which is known as a method with probabilistic data identification. The advantage of this method in comparison with the tracking method with identification at a minimum distance of the mark from the center of the gate is the fact that with the deterioration of the noise environment (an increase in the average number of false marks in the gate), the number of dropped false trajectories significantly increases. However, if there are four marks in the strobe, the complexity of method [16] doubles.

The methods for identifying radar information described above belong to the class of strobe methods with sequential data arrival. The methods are used mainly in solving problems of identifying single objects in conditions of interference. At the same time, when calculating the hypotheses of the origin of marks, the possibility of their belonging to another accompanied object is not taken into account. We are talking about the joint tracking of several objects moving at short distances from each other. This occurs when crossing their trajectories or when flying in a group. In these cases, the tracking strobes overlap with each other and the resulting markings can be correlated with multiple trajectories.

The problems of synthesizing methods for identifying objects in the presence of marks from closely located objects in the strobe were considered in [17-18]. The following methods can be distinguished as methods for identifying measurements obtained from a group of objects in conditions of interference:

- a tracking method based on a multi-hypothetical data identification model [17];

- a tracking method based on the model of joint probabilistic data identification [18].

The essence of method [17] is as follows. First, groups of objects with overlapping strobes are selected, and for these groups all possible options for elevation binding are generated, taking into account the entered a priori data on the sources of new elevations. The following hypotheses are accepted regarding the belonging of each mark:

- the mark belongs to one of the tracked trajectory included in the cluster; 


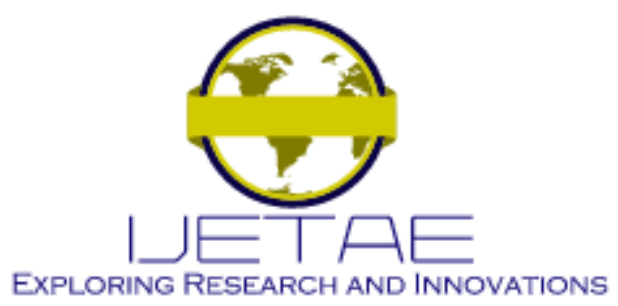

International Journal of Emerging Technology and Advanced Engineering

Website: www.ijetae.com (E-ISSN 2250-2459, Scopus Indexed, ISO 9001:2008 Certified Journal, Volume 11, Issue 12, December 2021)

- the mark belongs to the new object;

- the mark is formed by interference - false.

After that, the most plausible hypothesis is selected.

The advantages of method [17] are the higher quality of solving the identification problem when accompanying a group of objects in comparison with non-Bayesian algorithms. However, already in the presence of two tracked objects and three obtained during their tracking of marks, the number of options for binding measurements is equal to 30. This indicates a sufficient laboriousness of this method [17].

Method [18] involves the formation of joint hypotheses for identifying all measurements combined into a cluster and the objects being tracked. The main difference of method [18 is the formation of a class of so-called "background" objects. Background objects are hypotheses corresponding to the event when the flags hitting the strobe are false or belong to other objects. The creation of this class allows you to reduce the number of options for binding dimensions from thirty to eight.

Identification methods [17-18] belong to the class of Bayesian methods with parallel data input. These methods are characterized by lower values of indicators associated with erroneous identification, especially when tracking closely located objects. The disadvantages of methods [17-18] are the high labor intensity due to the formation and testing of a huge number of hypotheses, which makes these methods difficult to implement.

\section{MAterials AND RESEARCH MEthods}

We will use the measurement identification method, which belongs to the class of methods based on the Bayesian approach with parallel data input. In this case, the general formulation of the research problem can be formulated as follows. Observations are planned and carried out (measurement of current navigation parameters (MCNP)) for a group of space objects by a constellation of radio-technical observation equipment. The range of radiotechnical observation equipment is approximately 400$650 \mathrm{~km}$. It is assumed that the measurements obtained in one series of observations are independent and their errors are distributed according to the normal law with a zero mean and a correlation matrix of errors $\mathrm{K} 1$.
MCNP are received in series, taking into account the frequency of passage of objects through the observation zones of the observation facility. Each of the series of observations can contain both the results of observations of objects and background objects. Background objects are understood as all objects that do not belong to the class of maintained, and for which there is no information about their parameters and quantity. There is a priori information about the composition (quantity) and parameters of the tracked objects, which is contained in the object catalog. This information from the catalog of objects is an estimate of the motion parameters $\overline{\mathrm{m}}_{\mathrm{x}_{\mathrm{j}}}$ and a correlation matrix of errors $\mathrm{K}$ of the tracked object, obtained on the basis of previous observations.

It is also assumed that the parameters of motion of background objects are distributed according to a uniform law in the field of view of the observing facility. Considering the tactical and technical characteristics of the observation facility and the dynamics of the flight of objects during observation sessions, the objects of interest may be unsolvable. The task of the research is that, while receiving the ITNP during tracking, it is necessary to make a decision on whether specific measurements belong to specific objects from the group of interest or to a class of background objects. The processing of measurements obtained at different observation sessions is carried out sequentially when they are received, and the identification of measurements at one observation session is carried out jointly. This approach to solving the problem is due to the fact that the measurements obtained while accompanying the same objects, obtained at different observation sessions, are correlated with each other. That is, measurements are dependent [19].

We formulate the optimization problem for the indicated conditions as follows. It is necessary to find such a hypothesis for the joint identification of the tracked objects and the measurements obtained from them, which would provide a minimum value of the Bayesian average risk or a maximum of the Bayesian average payoff (1):

$$
B_{\text {med }}=\sum_{i=1}^{M} \sum_{j=1}^{M} P_{j} C_{i j} \int_{Z_{i}} P\left(\bar{x} / H_{j}\right) d \bar{x} \rightarrow \max ,
$$




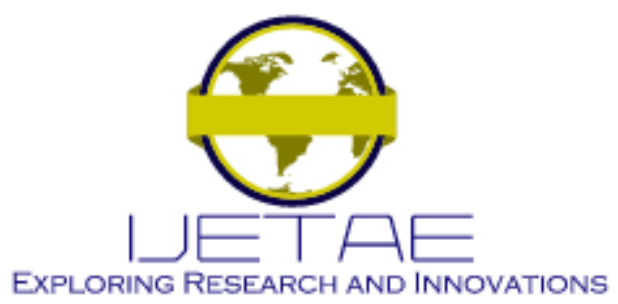

International Journal of Emerging Technology and Advanced Engineering

Website: www.ijetae.com (E-ISSN 2250-2459, Scopus Indexed, ISO 9001:2008 Certified Journal, Volume 11, Issue 12, December 2021)

where $\mathrm{P}_{\mathrm{j}}$ is the prior probability of accepting the hypothesis $\mathrm{H}_{\mathrm{j}}$;

$\mathrm{C}_{\mathrm{ij}}=1-\mathrm{B}_{\mathrm{ij}}$ are the elements of the matrix of reward for making the right decisions;

$B_{i j}$ are the elements of the matrix of losses for incorrectly made decisions;

$\mathrm{P}\left(\overline{\mathrm{x}} / \mathrm{H}_{\mathrm{j}}\right)$ is the conditional probability density of correct identification of an object, provided that a hypothesis $\mathrm{H}_{\mathrm{j}}$ is selected;

$\mathrm{Z}_{\mathrm{i}}$ is the area of the observation space from which the hypothesis $\mathrm{H}_{\mathrm{j}}$ is selected;

$\mathrm{M}$ is the number of hypotheses

under the following constraints (3):

$$
\left\{\begin{array}{l}
\sum_{j=1}^{N} \gamma_{k i j} \leq 1, \text { for } i=\overline{1, Q_{k}}, \\
\sum_{j=1}^{N} \gamma_{k 0 j} \leq N, \text { for } i=0,
\end{array}\right.
$$

where $\gamma_{k_{i j}}$ are the elements of the identification matrix;

$\mathrm{Q}_{\mathrm{k}}$ is the number of followed objects included in the $\mathrm{k}$-th group;

$\mathrm{N}$ is the number of measurements.

If the objects are allowed, the identification matrix $\gamma$ with elements $\gamma_{k_{i j}}$ has a dimension of (NxQ) (the index $\mathrm{k}$ for $\mathrm{Q}$ is omitted, since it is believed that we are talking about the k-th group of objects). An example of finding the indicated matrix is given in [19]. When objects are not resolved, the identification matrices have different dimensions depending on the options for not resolving objects. So, for example, for the case of three objects $(\mathrm{Q}=3)$, there are five different non-resolution options:

- all objects are resolvable;

- the first and second objects are not resolvable, the third is resolvable;

- the first and third objects are not resolvable, the second is resolvable;

- the third and second objects are not resolvable, the first is resolvable; - all objects are unresolvable.

Variants of non-resolution of objects for the case of three objects and the corresponding number of hypotheses are illustrated in Fig. 2.
To solve the identification problem in the case of nonresolution of objects, we divide the observation space $Z_{x}$ into disjoint subdomains $Z_{i}$, such that $Z_{x}=\sum_{i=1}^{M} Z_{i}$. At the same time, we will assume that the reward matrix for correctly made decisions is simple, with $\mathrm{C}_{\mathrm{ij}}=$ const, $\mathrm{Cij}=0$ [19]. Expression (2) can be rewritten as (4)

$$
\begin{aligned}
& B_{m e d}=\sum_{m=1}^{M} P_{m} C_{m m} \int_{Z_{m}} P\left(\bar{x} / H_{m}\right) d x= \\
& \sum_{m=1}^{M} P_{m} C_{m m} \int_{Z_{x}} \delta_{m}(\bar{x}) \cdot P\left(\bar{x} / H_{m}\right) d x
\end{aligned}
$$

where

$$
\delta_{m}(\bar{x})=\left\{\begin{array}{l}
1, \text { if } \bar{x} \in Z_{m} \\
0, \text { if } \bar{x} \notin Z_{m}
\end{array}\right.
$$

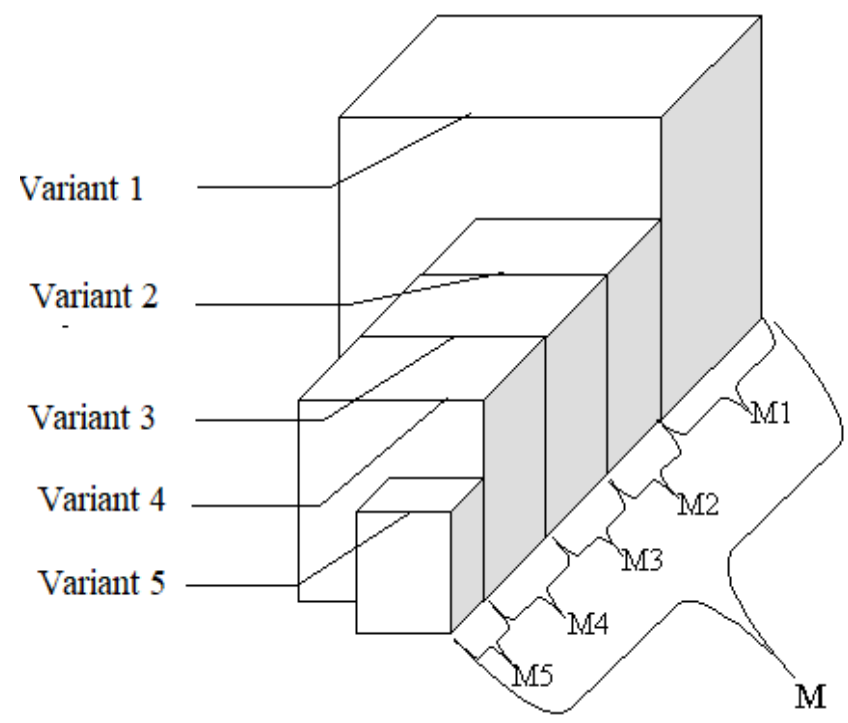

Fig. 2 The variants of non-resolution of objects for the case of three objects and the corresponding number of hypotheses

Assuming that the measurements are statistically independent from each other, we will calculate the joint distribution density of the sample values $\bar{x}$. We will accept the condition that they were generated by the objects included in the m-th hypothesis. 


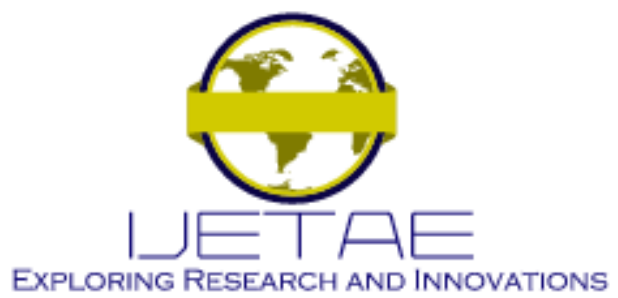

International Journal of Emerging Technology and Advanced Engineering

Website: www.ijetae.com (E-ISSN 2250-2459, Scopus Indexed, ISO 9001:2008 Certified Journal, Volume 11, Issue 12, December 2021)

The joint flatness of the distribution of sample values represents the product of the probability densities $\varphi_{\mathrm{i}}\left(\overline{\mathrm{x}_{\mathrm{i}}} / \mathrm{H}_{\mathrm{m}_{\mathrm{k}}}\right)$ of elements of the sample about the belonging of the $\mathrm{i}$-th dimension to the $\mathrm{j}$-th object included in the $\mathrm{m}$-th hypothesis, for the k-th variant of non-resolution related to this hypothesis, can be written in the form (5):

$$
P\left(\bar{x} / H_{m_{k}}\right)=\prod_{i=1}^{N} \phi_{i}\left(\overline{x_{i}} / H_{m_{k}}\right) .
$$

The probability density $\varphi_{\mathrm{i}}\left(\overline{\mathrm{x}_{\mathrm{i}}} / \mathrm{H}_{\mathrm{m}_{\mathrm{k}}}\right)$, for the case when the measurement is generated by a single object, can be represented in the form (6):

$$
\phi_{i}\left(\bar{x}_{i} / H_{m_{k}}\right)=\int_{-\infty}^{\infty} P\left(\bar{x}_{i} / \overline{\theta_{j m_{k}}}\right) \cdot P\left(\overline{\theta_{j m_{k}}}\right) d \overline{\theta_{j m_{k}}}, \text { (6) }
$$

where $P\left(\bar{x}_{i} / \overline{\theta_{j_{k}}}\right)$ is the conditional density of the distribution of measurement parameters at a point, provided that this measurement was generated by the $\mathrm{j}$-th object included in the $\mathrm{m}$-th hypothesis about the joint identification of objects and measurements on the k-th variant of non-resolution, with parameters;

$\mathrm{P}\left(\overline{\theta_{\mathrm{jm}_{\mathrm{k}}}}\right)$ is the prior distribution of the parameters of the $\mathrm{j}$ th object.

Assuming that the measurements are statistically independent, we will calculate the joint distribution density of sample values $\overline{\mathrm{x}}$. Let us take into account the condition that the measurements were generated by the objects included in the m-th hypothesis. Then the joint density of the distribution of the sample values is the product of the probability densities $\varphi_{\mathrm{i}}\left(\overline{\mathrm{x}_{\mathrm{i}}} / \mathrm{H}_{\mathrm{m}_{\mathrm{k}}}\right)$, the elements of the sample about the belonging of the $\mathrm{i}$-th dimension to the $\mathrm{j}$-th object included in the $\mathrm{m}$-th hypothesis, for the $\mathrm{k}$-th variant of non-resolution, also related to this hypothesis, can be written in the form (7):

$$
P\left(\bar{x} / H_{m_{k}}\right)=\prod_{i=1}^{N} \phi_{i}\left(\overline{x_{i}} / H_{m_{k}}\right)
$$

The probability density $\varphi_{\mathrm{i}}\left(\overline{\mathrm{x}_{\mathrm{i}}} / \mathrm{H}_{\mathrm{m}_{\mathrm{k}}}\right)$, for the case when this measurement was generated by a single object, can be represented in the following form (8):

$$
\phi_{i}\left(\bar{x}_{i} / H_{m_{k}}\right)=\int_{-\infty}^{\infty} P\left(\bar{x}_{i} / \overline{\theta_{j m_{k}}}\right) \cdot P\left(\overline{\theta_{j m_{k}}}\right) d \overline{\theta_{j m_{k}}},
$$

where $\mathrm{P}\left(\overline{\mathrm{x}_{\mathrm{i}}} / \overline{\theta_{\mathrm{j}_{\mathrm{k}}}}\right)$ is the conditional distribution density of measurement parameters $\overline{\mathrm{x}}$ at point $\overline{\mathrm{x}_{\mathrm{i}}}$, provided that this measurement was generated by the $j$-th object included in the m-th hypothesis of joint identification of objects and measurements on the k-th non-resolution option, with parameters $\theta_{\mathrm{jm}_{\mathrm{k}}}$;

$\mathrm{P}\left(\overline{\theta_{\mathrm{jm}_{\mathrm{k}}}}\right)$ is the prior distribution of the parameters of the $\mathrm{j}$ th object.

For the class of background objects, expression (9) is valid:

$$
\begin{aligned}
& \phi_{i}\left(\bar{x}_{i} / j=0\right)=\frac{1}{\Delta x} \cdot \int_{\Delta \bar{x}} \frac{1}{(\sqrt{2 \pi})^{n} \sqrt{\left\|K_{i j}\right\|}} \times \\
& \times \exp \left\{-\frac{1}{2}\left(\bar{x}_{i}-\bar{m}_{x \theta_{j m_{k}}}\right)^{T} \cdot K 1^{-1} \cdot\left(\bar{x}_{i}-\bar{m}_{x \theta_{j m_{k}}}\right)\right\} d \bar{m}_{x \theta_{j m_{k}}} \cong \\
& \cong \frac{1}{\Delta x} .
\end{aligned}
$$

In view of the above, expression (2) can be written in the form (10):

$$
B_{\text {med }}=\sum_{m=1}^{M_{k}} P_{m} C_{m_{k} m_{k}} \int_{Z_{x}} \delta_{m_{k}}(\bar{x}) \cdot \prod_{i=1}^{N} \varphi_{i}\left(\bar{x}_{i} / H_{m_{k}}\right) d \bar{x},
$$

and the optimization problem will look like this (11):

$$
\begin{aligned}
& B_{\text {med }}=\max _{\substack{\hat{\lambda} \\
\delta_{m_{k}}(\bar{x})}}\left\{\sum_{m=1}^{M_{k}} P_{m} C_{m_{k} m_{k}} \int_{Z_{x}} \delta_{m_{k}}(\bar{x}) \cdot \prod_{i=1}^{N} \varphi_{i}\left(\bar{x}_{i} / H_{m_{k}}\right) d \bar{x}\right\}= \\
& =\max _{\substack{\hat{\delta_{m_{k}}}(\bar{x}) \\
\hat{m}}}\left\{\int_{Z_{x}}\left[\sum_{m=1}^{M_{k}} P_{m} C_{m_{k} m_{k}} \delta_{m_{k}}(\bar{x}) \cdot \prod_{i=1}^{N} \varphi_{i}\left(\bar{x}_{i} / H_{m_{k}}\right) d \bar{x}\right]\right\} .
\end{aligned}
$$

Denoting the integrand in (11) by $\mathrm{L}_{\mathrm{k}}(\overline{\mathrm{x}})$ [19],

$$
L_{k}(\bar{x})=\sum_{m=1}^{M_{k}} L_{m_{k}}(\bar{x})=\sum_{m=1}^{M_{k}} C_{m_{k} m_{k}} P_{m_{k}} \prod_{i=1}^{N} \phi_{i}\left(\bar{x}_{i} / H_{m_{k}}\right),
$$

It is easy to show that $\delta_{m_{k}}(\bar{x})$ should be chosen equal to one if $\mathrm{L}_{\mathrm{m}_{\mathrm{k}}}(\overline{\mathrm{x}})$ is the largest for $\mathrm{m}_{\mathrm{k}}=\overline{1, \mathrm{M}_{\mathrm{k}}}$. 


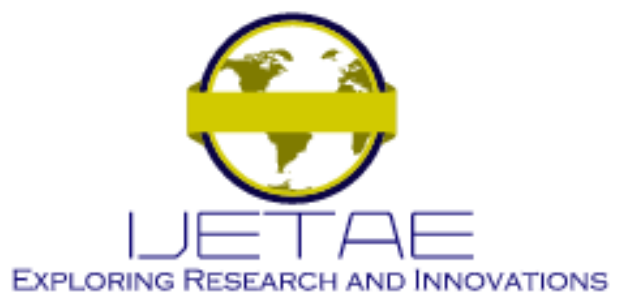

International Journal of Emerging Technology and Advanced Engineering

Website: www.ijetae.com (E-ISSN 2250-2459, Scopus Indexed, ISO 9001:2008 Certified Journal, Volume 11, Issue 12, December 2021)

Thus, taking into account (12), the solution to the optimization problem (11) is achieved by finding a hypothesis that provides a maximum of $\mathrm{L}_{\mathrm{m}_{\mathrm{k}}}\left(\frac{-}{\mathrm{x}}\right)$ (expression (13):

$$
\hat{m}_{k}=\arg \max \left\{L_{m_{k}}(\bar{x})\right\}, \quad \text { for } m_{k}=\overline{1, M_{k}} .
$$

Let us introduce matrix $\Phi_{\mathrm{m}_{\mathrm{k}}}=\left\{\varphi_{\mathrm{ij}} \mathrm{m}_{\mathrm{ij}}\right\}$, the elements of which represent the values of the likelihood function, provided that the i-th measurement was obtained while accompanying the $j$-th object or an unsolvable group of objects. The elements of this matrix can be calculated according to expression (7) - for single objects included in the catalog $\left(\mathrm{j}=\overline{1, \mathrm{Q}_{\mathrm{m}_{\mathrm{k}}}}\right)$, expression (9) - for an unsolvable group of objects. In this case, the elements of the matrix $\Phi_{m_{k}}$ for the class of background objects $(j=0)$ can be calculated from the expression (8).

Then the solution to the optimization problem, finding a hypothesis about the joint identification of objects and measurements on the kth variant of non-resolution (13), will take the form (14):

$$
\hat{m}_{k}=\arg \max \left\{C_{m_{k} m_{k}} P_{m_{k}} \cdot \prod_{i=1}^{N} \prod_{j=0}^{Q_{m_{k}}} \phi_{m_{k i j}}{ }^{\gamma_{m_{k i j}}}\right\},
$$

where $\gamma_{\mathrm{m}_{\mathrm{k}}}$ is the identification matrix corresponding to the m-th hypothesis;

$\mathrm{Q}_{\mathrm{m}_{\mathrm{k}}}$ is the number of allowed groups in the m-th hypothesis.

Problem (14), finding the hypothesis number $\hat{\mathrm{m}}_{\mathrm{k}}$, is equivalent to the problem of finding the identification matrix $\hat{\gamma}_{\mathrm{m}_{\mathrm{k}}}$ corresponding to the given hypothesis number.

Taking the logarithm of (14), we finally get (15):

$$
\hat{\gamma}_{m_{k}}=\arg \max \left\{\ln C_{m_{k} m_{k}}+\ln P_{m_{k}}+\sum_{i=1}^{N} \sum_{j=0}^{Q_{m_{k}}} \gamma_{m_{k i j}} \ln \phi_{m_{k i j}}\right\} \text {. }
$$

Expression (15) represents the optimal decision rule for the method of joint identification of radio engineering measurements while tracking closely spaced objects of observation. The optimal decision rule presupposes an enumeration of hypotheses.
Thus, the method of for identification of radars measurements of nearby objects tracking, in contrast to the known ones, assumes:

- solving the optimization problem of searching for a hypothesis of joint identification of objects and measurements;

- the solution is optimal according to Bayes' criterion;

- the solution of the optimization problem of joint identification of objects and measurements is reduced to the assignment problem with the subsequent choice of a hypothesis. The choice of hypothesis is presented in the form of an identification matrix;

- the presence of background objects in the viewing area is taken into account.

\section{CONCLUSIONS}

Thus, the paper proposes the method for identification of radars measurements of nearby objects tracking. The method for identification of radars measurements of nearby objects tracking, in contrast to the known ones, assumes. Solving the optimization problem of searching for a hypothesis of joint identification of objects and measurements. The solution is optimal according to Bayes' criterion. The solution of the optimization problem of joint identification of objects and measurements is reduced to the assignment problem with the subsequent choice of a hypothesis. The choice of hypothesis is presented in the form of an identification matrix. The presence of background objects in the viewing area is taken into account.

The procedure for enumerating hypotheses in accordance with rule (15) is rather cumbersome even with a small number of objects (10-12 objects). Therefore, the subject of further research is to find ways to reduce the computational costs of solving the optimization problem.

\section{REFERENCES}

[1] Khudov H., Diakonov O., Kuchuk N., Maliuha V., Furmanov K., Mylashenko I., Olshevskyi Y., Stetsiv S., Solomonenko Y., Yuzova I. Method for determining coordinates of airborne objects by radars with additional use of ADS-B receivers. Eastern-European Journal of Enterprise Technologies, № 4/9 (112), 2021. P. 54-64. DOI: https://doi.org/10.15587/1729-4061.2021.238407.

[2] Anttonen A., Kiviranta M., Höyhtyä M. Space debris detection over intersatellite communication signals. Acta Astronautica, Vol. 187, 2021. P. 156-166.

[3] Retrieved at: http://users2.ev1.net/ mmccants/tles/alldat.zip. 


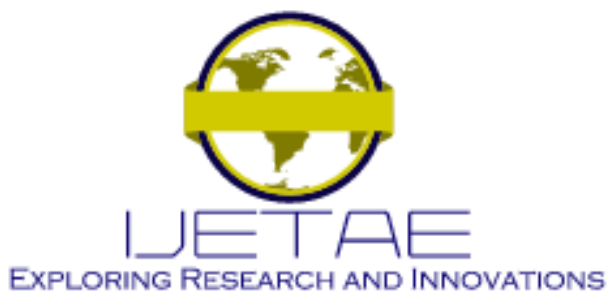

International Journal of Emerging Technology and Advanced Engineering

Website: www.ijetae.com (E-ISSN 2250-2459, Scopus Indexed, ISO 9001:2008 Certified Journal, Volume 11, Issue 12, December 2021)

[4] Retrieved at: http://users2.ev1.net/ mmccants/tles/index.html.

[5] Fengler A., Haghighatshoar S., Jung P., Caire G. Non-Bayesian Activity Detection, Large-Scale Fading Coefficient Estimation, and Unsourced Random Access With a Massive MIMO Receiver. IEEE Transactions on Information Theory. Vol. 67, Issue 5, 2021. P. 2925 - 2951. DOI: https://doi.org/0.1109/TIT.2021.3065291

[6] Tu. G., Chen C., Gong Z., Wang Y. A Form-Finding Method for Branching Structures Based on Dynamic Relaxation. Applied Science. $\quad$ № 11ю 2021. $7165 . \quad$ DOI: https://doi.org/10.3390/app11157165.

[7] Faul A. C., Tipping M. E. Analysis of sparse Bayesian learning. In T. G. Dietterich, S. Becker, and Z. Ghahramani (Eds.), Advances in Neural Information Processing Systems 14, 2002. P. 383-389. MIT Press.

[8] Khudov H., Zvonko A., Khizhnyak I., Shulezko V., Khlopiachyi V., Chepurnyi V., Yuzova I. The Synthesis of the Optimal Decision Rule for Detecting an Object in a Joint Search and Detection of Objects by the Criterion of Maximum Likelihood, International Journal of Emerging Trends in Engineering Research, № 8(2), 2020, P. 520-524. DOI: https://doi.org/10.30534/ijeter/2020/40822020.

[9] Khudov H., Khizhnyak I., Zots F., Misiyuk G., Serdiuk O. The Bayes Rule of Decision Making in Joint Optimization of Search and Detection of Objects in Technical Systems, International Journal of Emerging Trends in Engineering Research, № 8(1), 2020, P. 7-12. DOI: https://doi.org/10.30534/ijeter/2020/02812020.

[10] Khudov G.V. Features of optimization of two-alternative decisions by joint search and detection of objects. Problemy Upravleniya I Informatiki (Avtomatika), 2003, № 5, P. 51-59.

[11] Bar-Shalom Y. Tracking methods in a multitarget environment. IEEE Trans. on automatic control. Vol. 23. № 4. 1978. P. 618-626.
[12] Kuo-Chu Chang, Bar-Shalom Y. Join probabilistic date association for multitarget tracking whis possibly unresolved measurements and maneuvers. IEEE Trans. on AC. Vol. 29. № 7. 1984. P. 585-594.

[13] Forthmann T.E., Bar-Shalom Y., Scheffe M.. Sonar tracking of multiple targets using joint probabilistic date association. IEEE Journal of oceanic engineering. Vol. 8. № 3. 1983. P. 173-193.

[14] Khudov H., Khizhnyak I., Koval V., Maliuha V., Zvonko A., Yunda V., Nagachevskyi V., Berezanskyi V. The Efficiency Estimation Method of Joint Search and Detection of Objects for Surveillance Technical Systems, International Journal of Emerging Trends in Engineering Research, Vol. 8. № 3, 2020, P. 813-819.

[15] LeRoy T.E., Hayden B.L., Desmarais J., Menendez M.E., War D. Early Outcome Comparison of the Posterior Approach and the Superior Approach for Primary Total Hip Arthroplasty. Arthroplasty Today. Vol. 6, Iss. 3, 2020, $\quad$ P. 508-512. DOI: https://doi.org/10.1016/j.artd.2020.05.005.

[16] Inoue A., Shintani M. Quasi-Bayesian Model Selection. 2020. Retrieved at: https://www.qeconomics.org/ojs/forth/587/587-3.pdf.

[17] Meinhardt T., Leal-Taixe L., Bergmann P. Tracking without bells and whistles. 2019. Retrieved at: https://arxiv.org/pdf/1903.05625.pdf

[18] Shaoming He S., Shin H.S., Tsourdos A. Distributed multiple model joint probabilistic data association with Gibbs sampling-aided implementation. Information Fusion. Vol. 64, 2020, P. 20-31. DOI: https://doi.org/10.1016/j.inffus.2020.04.007

[19] Khudov H., Yarosh S., Savran V., Zvonko A.,, Shcherba A., Arkushenko P. The Technique of Research on the Development of Radar Methods of Small Air Objects Detection, International Journal of Emerging Trends in Engineering Research, Vol. 8. № 7, 2020, pp. 3708-3715.

https://doi.org/10.30534/ijeter/2020/132872020. 